\title{
A novel Parameter Estimation method based on FRFT in Bistatic MIMO Radar System
}

\author{
Li Li $i^{1, a}$ \\ ${ }^{1}$ Information Engineering College, Dalian University, China \\ affsimple@163.com
}

Keywords: bistatic MIMO radar; FRFT; Doppler stretch factor; Time delay; DOD-DOA

\begin{abstract}
We study the problem of parameter estimation in in wideband bistatic MIMO radar system. Firstly, we propose a novel algorithm to estimate Doppler stretch and time delay in fractional Fourier transform (FRFT) domain. Secondly, we also construct two sub-array models based on the peaks of fractional Fourier transform (FRFT) to accurately estimate the direction-of-departure (DOD) and the direction-of-arrival (DOA). Furthermore, the Cramér-Rao bound for parameter estimation is derived and computed in closed form. Parameter estimation performances are evaluated and studied theoretically and via simulations. The simulation results demonstrate that the proposed method can get better estimation accuracy.
\end{abstract}

\section{Introduction}

Multiple-Input Multiple-Output (MIMO) system has attracted more and more attention for its ability to enhance system performance ${ }^{[1,2]}$. A MIMO radar system consists of both transmit and receive sensors, with the transmit sensors having the ability to transmit orthogonal waveforms. However, these existing methods have certain limitations. Qu [3] estimates time delay and Doppler stretch for wideband signals based on the wideband ambiguity function. Ma [9] proposes two novel methods for broadband chirp DOA estimation, These methods obtain good performance for DOA estimation of wideband signal, however, they did not estimate the Doppler and time delay which are also very crucial for the determination of the range and velocity of the target in wideband bistatic MIMO radar. At present, we seldom find the study of joint estimation for Doppler, time delay, DOD and DOA in wideband bistatic MIMO radar, which should be studied deeply for target tracking and target localization. So, in this paper, we study parameter estimation of wideband signal model for multiple targets localization in the context of wideband bistatic MIMO radar.

\section{The Proposed Signal Model}

We consider a bistatic MIMO radar system with $Q$ closely spaced transmit antennas and $N$ closely spaced receive antennas. $d_{\mathrm{t}}$ and $d_{\mathrm{r}}$ are interelement spacing at the transmitter and the receiver respectively. For improving the ability of anti-interference, each transmit antenna transmits chirp signal $x_{q}(t)=A_{q} \exp \left(j 2 \pi\left(f_{q 0} t+\mu_{q 0} t^{2} / 2\right)\right)$ for $q=1, \ldots, Q .\left(\varphi_{l}, \theta_{l}\right)$ for $l=1, \ldots, L$, where $\varphi_{l}$ denotes the DOD and $\theta_{l}$ denotes the DOA. The received signal $s(t)$, reflected from $L$ moving targets, can be expressed as

$$
\boldsymbol{s}(t)=\sum_{l=1}^{L} \beta_{l} \boldsymbol{x}\left(a_{l}\left(t-\tau_{l}\right)\right) \boldsymbol{A}\left(\varphi_{l}\right) \boldsymbol{B}^{\mathrm{T}}\left(\theta_{l}\right)+\boldsymbol{n}(t)
$$

where $\beta_{l}$ denotes the Radar Cross-Section, $a_{l}$ and $\tau_{l}$ denote the Doppler stretch factor and the time delay, $\quad \boldsymbol{x}\left(a_{l}\left(t-\tau_{l}\right)\right)=\left[x_{1}\left(a_{l}\left(t-\tau_{l}\right)\right), \ldots, x_{Q}\left(a_{l}\left(t-\tau_{l}\right)\right)\right]^{\mathrm{T}}$, where $[\cdot]^{\mathrm{T}}$ denotes the transpose matrix. $\boldsymbol{A}\left(\varphi_{l}\right)=\left[A_{1}\left(\varphi_{l}\right), \ldots, A_{Q}\left(\varphi_{l}\right)\right]$ and $\boldsymbol{B}\left(\theta_{l}\right)=\left[B_{1}\left(\theta_{l}\right), \ldots, B_{N}\left(\theta_{l}\right)\right]$, where $A_{q}\left(\varphi_{l}\right)=\exp \left(j 2 \pi(q-1) d_{\mathrm{t}} \sin \varphi_{l} / \lambda\right)$ 
and $B_{n}\left(\theta_{l}\right)=\exp \left(j 2 \pi(n-1) d_{\mathrm{r}} \sin \theta_{l} / \lambda\right) . \boldsymbol{n}(t)$ is assumed to be independent, zero-mean Gaussian white noise with variance $\sigma_{w}^{2}$.

\section{FRFT analysis of the Proposed Signal Model}

The FRFT of a signal $f(t)$ with an angle $\alpha$ is defined as

$$
F(\alpha, m)=F^{p}[f(t)](m)=\int_{-\infty}^{+\infty} f(t) K_{p}(t, m) d t
$$

where $F^{p}$ denotes the FRFT operator, $p$ for $0<p \leq 2$ is the order of the FRFT, and $K_{p}(t, m)$ is the kernel function of the fractional Fourier transform. $K_{p}(t, m)$ can be expressed as

$$
K_{p}(t, m)= \begin{cases}A_{\alpha} \exp \left(j \pi\left(t^{2} \cot \alpha-2 m t \csc \alpha+m^{2} \cot \alpha\right)\right), \alpha \neq n \pi \\ \delta(t-m), & \alpha=2 n \pi \\ \delta(t+m), & \alpha=(2 n+1) \pi\end{cases}
$$

Where $A_{\alpha}=\sqrt{(1-j \cot \alpha)} \alpha \equiv p \pi / 2, m$ is the fractional Fourier frequency and $n$ is an integer.

\section{FRFT of chirp signal}

According to (2) and (3), the FRFT of chirp signal $x_{q}(t)$ with an angle $\alpha$ is defined as

$$
X_{q}(\alpha, m)=A_{q} A_{\alpha} \exp \left(j \pi m^{2} \cot \alpha\right) \int_{0}^{T} \exp \left(j 2 \pi t\left(f_{q 0}-m \csc \alpha\right)\right) \exp \left(j \pi t^{2}\left(\cot \alpha+\mu_{q 0}\right)\right) d t \text {. }
$$

When $\alpha_{q 0}=-\operatorname{arccot}\left(\mu_{q 0}\right)$ and $m_{q 0}=f_{q 0} \sin \alpha_{q 0}, X_{q}\left(\alpha_{q 0}, m\right)$ could achieve its peak at $\left(\alpha_{q 0}, m_{q 0}\right)$. Therefore, we can obtain $\mu_{q 0}=-\cot \alpha_{q 0}$ and $f_{q 0}=m_{q 0} \csc \alpha_{q 0}$. We can conclude that the rotation angle $\alpha$ is only associated with the chirp rate of the signal.

\section{Matched filtering in FRFT domain}

The $q$ th band-pass matched filter with suitable bandwidth and central frequency $m_{q 0}$ is designed. Let $R_{q, n}(\alpha, m)$ denote the output of the $q$ th matched filter at the $n$th receive antenna, and $R_{q, n}(\alpha, m)=F^{p}\left[r_{q, n}(t)\right](m)$, where $r_{q, n}(t)=\sum_{l=1}^{L} \beta_{l} x\left(a_{l}\left(t-\tau_{l}\right)\right) A_{q}\left(\varphi_{l}\right) B_{n}\left(\theta_{l}\right)+\boldsymbol{w}(t), \boldsymbol{w}(t)$ is the output noise of the filter in the time domain which could still be approximately regarded as a white Gaussian noise.

\section{Parameter Estimation based on the FRFT}

\section{Joint DS and TD Estimation.}

We can obtain that Equation $R_{q, n}(\alpha, m)$ could achieve its peak at $\left(\alpha_{q l}, m_{q l}\right)$, and its peak value is $\left|\beta_{l}\right| A_{q} T A_{\alpha_{q l}} \exp \left(j \pi m_{q l}^{2} \cot \alpha_{q l}\right)$. According to the relation between $\left(\alpha_{q l}, m_{q l}\right)$ and $\left(f_{q l}, \mu_{q l}\right)$, Doppler stretch factor $a_{l}$ and time delay $\tau_{l}$ are obtained by

$$
\left\{\begin{array}{l}
a_{l}=\sqrt{-\cot \alpha_{q l} / \mu_{q 0}} \\
\tau_{l}=\left(f_{q 0} a_{l} \sin \alpha_{q l}-m_{q l}\right) /\left(\mu_{q 0} a_{l}^{2} \sin \alpha_{q l}\right)
\end{array}\right.
$$

\section{Joint DOD and DOA Estimation.}

We can define $y_{q, l}(t)=A_{q} \exp \left(j 2 \pi\left(f_{q l} t+\mu_{q l} t^{2} / 2\right)\right) . Y_{q, l}(\alpha, m)$ could achieve its peak at $\left(\alpha_{q l}, m_{q l}^{\prime}\right)$, and its peak value is $Y_{q, l}\left(\alpha_{q l}, m_{q l}\right)=A_{q} A_{\alpha_{q l}} \exp \left(j \pi m_{q l}{ }^{2} \cot \alpha_{q l}\right) \cdot T$.

According to their peak vlues $R_{q, n}(\alpha, m)$ and $Y_{q, l}(\alpha, m)$, we can obtain as 


$$
R_{q, n}(\alpha, m)=\sum_{l=1}^{L} R_{q, n, l}\left(\alpha_{q l}, m_{q l}\right)+W(\alpha, m)=\sum_{l=1}^{L} C_{q, n, l}(\varphi, \theta) \cdot Y_{q, l}\left(\alpha_{q l}, m_{q l}^{\prime}\right)+W(\alpha, m)
$$

where $C_{q, n, l}(\varphi, \theta)=h_{q, n, l}(\varphi, \theta) \exp \left(j \pi\left(\left(\tau_{l} \cos \alpha_{q l}\right)^{2}+2 m_{q l}^{\prime} \tau_{l} \cos \alpha_{q l}\right) \cot \alpha_{q l}\right)$.

At $\left(\alpha_{q l}, m_{q l}\right)$, we can get $R_{q, n}\left(\alpha_{q l}, m_{q l}\right)=R_{q, n, l}\left(\alpha_{q l}, m_{q l}\right)+\sum_{\eta \neq l}^{L} R_{q, n, \eta}\left(\alpha_{q l}, m_{q l}\right)+W\left(\alpha_{q l}, m_{q l}\right)$. Selecting the data of $L$ peak points $R_{q, n, l}\left(\alpha_{q l}, m_{q l}\right)$ as observed data at the receiver, the output of the $n$th receive antenna in the FRFT domain can be expressed as $\boldsymbol{R}_{q, n}=\left[\begin{array}{llll}R_{q, n, 1}\left(\alpha_{q 1}, m_{q 1}\right) & \cdots & R_{q, n, L}\left(\alpha_{q L}, m_{q L}\right)\end{array}\right]$.

The output vector of the receiver can be modeled as $\boldsymbol{R}=\boldsymbol{C Y}+\boldsymbol{N}$, where $\boldsymbol{R}=\left[\begin{array}{lll}\boldsymbol{R}_{q, 1} & \cdots & \boldsymbol{R}_{q, N}\end{array}\right]^{\mathrm{T}}$, $\boldsymbol{C}=\left[\begin{array}{lll}\boldsymbol{C}_{q, 1} & \ldots & \boldsymbol{C}_{q, L}\end{array}\right], \boldsymbol{Y}=\operatorname{diag}\left\{Y_{q, 1}\left(\alpha_{q 1}, m_{q 1}^{\prime}\right) \quad \ldots \quad Y_{q, L}\left(\alpha_{q L}, m_{q L}^{\prime}\right)\right\}, \quad \boldsymbol{C}_{q, l}=\left[\begin{array}{llll}C_{q, 1, l} & C_{q, 2, l} & \ldots & C_{q, N, l}\end{array}\right]^{T}, \operatorname{diag}(\cdot)$ denotes a diagonal matrix .

Both receive subarrays are constructed in FRFT domain as follows $\boldsymbol{R}_{1}=\boldsymbol{F} \boldsymbol{Y}+\boldsymbol{N}_{1} \quad q=1$ and $\boldsymbol{R}_{2}=\boldsymbol{F} \boldsymbol{G} \boldsymbol{Y}+\boldsymbol{N}_{2} \quad q \neq 1 \quad$, where $\quad \boldsymbol{G}=\operatorname{diag}\left\{\exp \left(j 2 \pi(q-1) \rho_{q} \sin \varphi_{1}\right), \ldots, \exp \left(j 2 \pi(q-1) \rho_{q} \sin \varphi_{L}\right)\right\} \quad$, $\boldsymbol{F}=\left[\begin{array}{llll}\boldsymbol{C}_{1,1} & \boldsymbol{C}_{1,2} & \ldots & \boldsymbol{C}_{1, L}\end{array}\right], C_{q, n, l}(\varphi, \theta)=h_{q, n, l}(\varphi, \theta) \exp \left(j \pi\left(\left(\tau_{l} \cos \alpha_{q l}\right)^{2}+2 m_{q l}^{\prime} \tau_{l} \cos \alpha_{q l}\right) \cot \alpha_{q l}\right)$.

DOA Estimation based on FRFT-MUSIC . As the signal $Y$ is independent of the noise, the correlation matrix $\boldsymbol{R}_{R_{1} R_{1}}$ of the subarray $\boldsymbol{R}_{1}$ can be expressed as $\boldsymbol{R}_{R_{1} R_{1}}=\boldsymbol{F} \boldsymbol{R}_{Y Y} \boldsymbol{F}^{\mathrm{H}}+\sigma_{n}^{2} \boldsymbol{I}$, where $[\cdot]^{\mathrm{H}}$ denotes the Hermitian transpose, $\boldsymbol{R}_{Y Y}$ is the signal covariance matrix and $\boldsymbol{I}$ is the unit matrix. Spatial spectrum of FRFT-MUSIC in fractional Fourier domain can be got based on classical MUSIC algorithm, which can be expressed as $P(\boldsymbol{\theta})=1 / \boldsymbol{F}^{\mathrm{H}}(\boldsymbol{\theta}) \boldsymbol{U}_{N} \boldsymbol{U}_{N}^{\mathrm{H}} \boldsymbol{F}(\boldsymbol{\theta})$. Searching spectral peak of $P(\boldsymbol{\theta})$, we can get the DOA estimator $\boldsymbol{\theta}$.

DOD Estimation based on FRFT-ESPRIT. Let $\boldsymbol{J}_{11}=\boldsymbol{R}_{R_{1} R_{1}}-\sigma^{2} \boldsymbol{I}=\boldsymbol{F} \boldsymbol{R}_{\mathrm{YY}} \boldsymbol{F}^{\mathrm{H}}$ and $\boldsymbol{J}_{12}=\boldsymbol{R}_{R_{1} R_{2}}-\sigma^{2} \boldsymbol{Z}$ $=\boldsymbol{F} \boldsymbol{G} \boldsymbol{R}_{Y Y} \boldsymbol{F}^{\mathrm{H}}$, where $\mathbf{Z}=[0 \ldots 0 ; 10 \ldots 0 ; \ldots ; 0 \ldots 10]$. Therefore, based on FRFT- ESPRIT algorithm, the DOD estimator $\varphi_{l}$ is obtained by $\varphi_{l}=\arcsin \left(\lambda_{q}^{\prime} \arg \left(g_{l}\right) / 2(q-1) d_{\mathrm{t}} \pi\right)$, where $g_{l}$ is the element of the principal diagonal of matrix $\boldsymbol{G}, \arg \left(g_{l}\right)$ stands for the phase of $g_{l}$.

\section{Cramér-Rao Bound}

The proposed wideband signal model $\boldsymbol{r}(t)$ can also be expressed as $\boldsymbol{r}(t)=\boldsymbol{K}(\boldsymbol{a}, \boldsymbol{\tau}, \boldsymbol{\varphi}, \boldsymbol{\theta}) \boldsymbol{\beta}+\boldsymbol{N}(t)$, where $\boldsymbol{K}(\boldsymbol{a}, \tau, \boldsymbol{\varphi}, \boldsymbol{\theta})=\left[\boldsymbol{k}\left(a_{1}, \tau_{1}, \varphi_{1}, \theta_{1}\right), \ldots, \boldsymbol{k}\left(a_{L}, \tau_{L}, \varphi_{L}, \theta_{L}\right)\right], \quad \boldsymbol{k}\left(a_{l}, \tau_{l}, \varphi_{l}, \theta_{l}\right)=\left[\boldsymbol{x}\left(a_{l}, \tau_{l}\right) \circ \boldsymbol{A}\left(\varphi_{l}\right)\right] \otimes \boldsymbol{B}\left(\theta_{l}\right)$, $\boldsymbol{A}(\boldsymbol{\varphi})=\left[\boldsymbol{A}\left(\varphi_{1}\right), \ldots, \boldsymbol{A}\left(\varphi_{L}\right)\right], \boldsymbol{B}(\boldsymbol{\theta})=\left[\boldsymbol{B}\left(\theta_{1}\right), \ldots, \boldsymbol{B}\left(\theta_{L}\right)\right], \otimes$ is Kronecker matrix product.

Suppose that the number of snapshots is $N_{s}$. In this case, element $i, j$ of the Fisher information matrix (FIM) for the $N_{s}$ observations can be shown to be equal to[6]

$$
\Gamma_{i j}(\xi)=2 \operatorname{Re} \sum_{t=1}^{N}\left\{\left(\frac{\partial \boldsymbol{K}(\boldsymbol{a}, \tau, \boldsymbol{\varphi}, \boldsymbol{\theta}) \boldsymbol{\beta}}{\partial \boldsymbol{\xi}_{i}}\right)^{\mathrm{H}} \boldsymbol{Q}_{n}^{-1}\left(\frac{\partial \boldsymbol{K}(\boldsymbol{a}, \tau, \boldsymbol{\varphi}, \boldsymbol{\theta}) \boldsymbol{\beta}}{\partial \boldsymbol{\xi}_{j}}\right)\right\}
$$

Since $\boldsymbol{K}(\boldsymbol{a}, \tau, \boldsymbol{\varphi}, \boldsymbol{\theta}) \boldsymbol{\beta}$ and $\boldsymbol{Q}_{n}$ depend on different elements of $\boldsymbol{\xi}$, it is clear that FIM will be block diagonal with respect to the signal $\boldsymbol{\xi}$ and noise $\boldsymbol{Q}_{n}=\sigma_{w}^{2} I_{N}$ parameters. SNR is defined as $\operatorname{SNR}=|\beta|^{2} N_{s} / \sigma_{w}^{2}$. The expression for the CRB is shown as $\mathbf{C R B}(\xi)=\Gamma^{-\mathbf{1}}$.

\section{Simulation results}

The considered bistatic MIMO radar is composed of $Q=2$ and $N=6$ with an interelement spacing of $0.5 \mathrm{~m}$. The number of Monte Carlo iterations is 500 in all simulations. 


\section{Simulation 1: Signal to Noise Ratio}

Fig.1 demonstrate the performance of the proposed method and the other methods versus SNR when $M=1200$.

\section{Simulation 2: Detection Performance}

In this simulation, $D=100 \mathrm{~km}$ is the base line distance between the transmit reference element and the receive reference element, and the targets is located at the angle $(\varphi, \theta)=\left(30^{\circ}, 80^{\circ}\right)$. We discuss the relationship target localization performance with the errors of the DOD and the DOA according to the literature [4] in Fig.2.

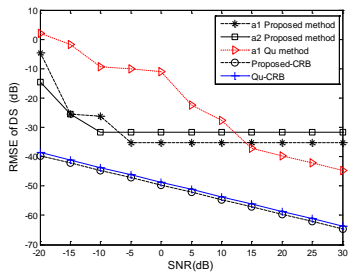

(a)

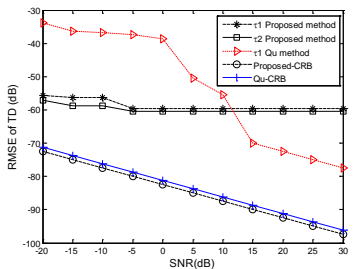

(b)

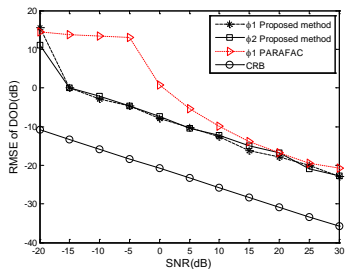

(c)

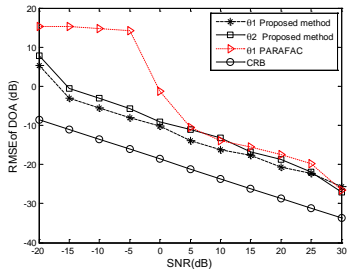

(d)

Fig.2 RMSE of DS estimation (a), TD estimation (b) DOD (c) and DOA (d) versus SNR

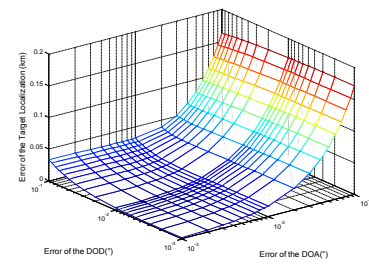

(a)

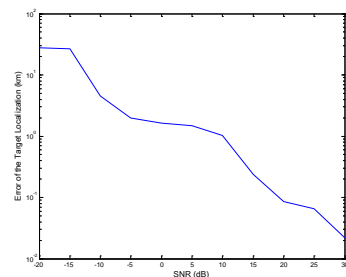

(b)

Fig.3 Error of the target localization

(a) Error of the target localization versus errors of the DOD and DOA, (b) Error of the target localization versus SNR

From Fig.3 (a), we obtain that the error of target localization increases versus errors increment of the DOD and the DOA. When the errors of the DOD and DOA are both $0.1 \pi / 180$ radians, the error of target localization is $171 \mathrm{~m}$. From Fig.3 (b), we find that the proposed method has good performance of target localization.

\section{Conclusion}

In this paper, we proposed a novel method for estimating mutil-targets parameters in wideband bistatic MIMO radar system. Firstly, time delay and Doppler stretch are estimated by searching peak of the FRFT function. Then, we accurately estimate the DOD and the DOA by employing the FRFT-MUSIC algorithm and the FRFT-ESPRIT algorithm. Furthermore, we derived the Cramér-Rao bound for target parameter estimation in wideband signal model. Simulation results demonstrate that the proposed method still has good performance with low SNR.

\section{Acknowledgments}

This work was partly supported by the National Science Foundation of China under Grants 61401055.

\section{References}

[1] H. Hao, P. Stocia, J. Li. Wideband MIMO systems: Signal Design for Transmit Beampattern synthesis, IEEE transactions on Signal Processing, 59 (2) (2011), p. 618-628

[2] P. Stoica, J. Li, Y. Xie, On Probing Signal Design For MIMO Radar, IEEE Transactions on Signal Processing 55 (8) (2007), p.4151-4161.

[3] J. Qu, M.W. Kon, and Z.Q. Luo, The estimation of time delay and Doppler stretch of wideband signals, IEEE Transactions on Signal Processing, 43 (4) (1995), p. 904-916. 
[4] B. Luis, Almeida. The fractional Fourier Transform and Time-Frequency Representations. IEEE Trans Signal Process 42(11),(1994), p. 3084-3091.

[5] H.M. Ozaktas, O. Arikan, M.A. Kautay. Digital Computation of the Fractional Fourier Transform. IEEE Transactions on Signal Processing, 44(9),(1996), p. 2141-2150.

[6] A.L. Swindlehurst, P. Stoica. Maximum Likelihood Methods in Radar Array signal processing. Proceedings of the IEEE, 86(2), (1998), p. 421-441. 